\title{
Establishment of hypoxia induction in an in vivo animal replacement model for experimental evaluation of pancreatic cancer
}

\author{
NATHALIE BAUER, LI LIU, EWA ALEKSANDROWICZ and INGRID HERR
}

\begin{abstract}
Molecular Oncosurgery, Department of General and Transplantation Surgery, University of Heidelberg and German Cancer Research Center (DKFZ), D-69120 Heidelberg, Germany
\end{abstract}

Received March 20, 2014; Accepted April 22, 2014

DOI: $10.3892 /$ or.2014.3196

\begin{abstract}
Transplantation of tumor xenografts to fertilized chicken eggs is a promising animal replacement method, which has successfully been used for xenotransplantation of pancreatic ductal adenocarcinoma (PDA) cells. PDA is characterized by a pronounced tumor hypoxia, which mediates aggressive growth, therapy resistance and cancer stem cell (CSC) features. For in vivo experimental evaluation of hypoxia-targeting therapeutic strategies, the xenografting of tumors to chicken eggs combined with the induction of hypoxia is necessary. However, the chicken embryos do not survive the conventional method of hypoxia induction by a gas mixture of $1 \% \mathrm{O}_{2}, 5 \% \mathrm{CO}_{2}, 94 \% \mathrm{~N}_{2}$, not even when hypoxia is applied for only $30 \mathrm{~min}$. Therefore, we employed chemical induction of hypoxia by the hypoxia mimetic agent cobalt chloride $\left(\mathrm{CoCl}_{2}\right)$. Whereas $\mathrm{CoCl}_{2}$ did not further increase tumor growth, it mediated the induction of carbonic anhydrase IX (CAIX) in the tumor xenografts and led to enhanced expression of the human CSC markers CD133, Sox2 and CD44. Side-effects in chicken embryos were not observed as evaluated by H\&E staining of embryo-derived liver sections and the determination of the embryo weight. These results suggest the successful induction of hypoxia in chicken eggs and xenografted tumors by $\mathrm{CoCl}_{2}$. For therapeutic intervention and as a control, we treated the eggs with the plant-derived anti-inflammatory agent triptolide, which recently showed promising effects toward hypoxiainduced tumor progression in experimental PDA. Triptolide abolished tumor growth and the $\mathrm{CoCl}_{2}$-induced hypoxic effects, without inducing obvious side-effects. Collectively,
\end{abstract}

Correspondence to: Professor Ingrid Herr, Experimental Surgery, Im Neuenheimer Feld 365, D-69120 Heidelberg, Germany

E-mail: i.herr@dkfz.de

Abbreviations: CAM, chorioallantoic membrane; CSC, cancer stem cell; EMT, epithelial-mesenchymal transition; $\mathrm{CoCl}_{2}$, cobalt chloride; PDA, pancreatic ductal adenocarcinoma

Key words: pancreatic cancer, chorioallantoic membrane, hypoxia, epithelial-mesenchymal transition, cancer stem cells our data present a new in vivo animal replacement method for the successful induction of tumor hypoxia in PDA.

\section{Introduction}

Although considerable advances in scientific knowledge and technology have been made, the use of animals in research is considered essential for understanding the disease mechanisms and evaluating the safety and efficacy of potential new therapeutic strategies. For animal welfare, different strategies for replacement, refinement and reduction of animals in scientific experiments have been suggested. For reduction and replacement of tumor transplantation on immunodeficient mice, we recently established the method of tumor transplantation on fertilized chicken eggs in our laboratory (1). According to the German animal law, the chick embryo is not considered a living animal, and thus is a unique model that overcomes many limitations to studying the biology of cancer in vivo. Similarly, the American Institutional Animal Care and Use Committee (IACUC) allows the use of the chick embryo for research experiments without the submission of an animal application until day 18 of development. The chorioallantoic membrane (CAM), a well-vascularized extra-embryonic tissue located underneath the eggshell, has a successful history as a biological platform for the molecular analysis of cancer $(2,3)$, although it is not yet generally accepted. The chick embryo is naturally immunodeficient and the CAM readily supports the engraftment of both normal and tumor tissues (4). According to our experience, human freshly resected tumor tissue grows even faster on eggs compared to immunodeficient mice. In addition to tumor growth, the CAM successfully supports the development of a characteristic tumor microenvironment and the detection of invasion, migration and angiogenesis. Other advantages are the complete environment in vivo, the possibility to easily study therapeutic effects to tumor take, growth and expression of signaling molecules along with side-effects, since the response of CAM tissue and chick embryo is similar to the mammalian response. A further advantage is the price and easy handling, since one fertilized chicken egg costs 25 cents and an animal house or a special room are not necessary. Tumor transplantation to eggs can be performed in a normal laboratory room equipped with small- 
sized breeding incubators. Therefore, we routinely use this animal replacement model for short-term in vivo evaluation of new therapeutic options for pancreatic ductal adenocarcinoma (PDA).

PDA is the most common type of pancreatic malignancy and it is usually diagnosed at an advanced state due to its untypical symptoms and aggressive behavior. PDA is characterized by an intense tumor stroma with pronounced hypoxic microenvironment (5), extensive local invasion, early systemic dissemination and resistance to chemo- and radiotherapy. The hypoxic microenvironment results in high expression of the hypoxia marker CAIX and the hypoxia-inducible factor HIF-1 $\alpha$ in patient tissue with poor clinical outcome (6). In experimental studies, hypoxia indicates aggressive growth and spontaneous metastasis in pancreatic cancer xenografts (7). Most importantly, hypoxia is supposed to promote invasion and metastasis and is involved in enrichment of cancer stem cells (CSCs).

To study if new therapeutic options can overcome the pronounced tumor hypoxia of PDA, a method for induction of hypoxia in fertilized chicken eggs is urgently needed. Since our preliminary results suggest that the conventional in vitro method of gas hypoxia using $1 \% \mathrm{O}_{2}, 5 \% \mathrm{CO}_{2}$ and $94 \% \mathrm{~N}_{2}$ is not suited for the chicken model, we employed chemical induction of hypoxia by cobalt chloride $\left(\mathrm{CoCl}_{2}\right)$. Previous publications have shown that exposure to cobalt promotes a response similar to hypoxia due to the stabilization of HIF-1 $\alpha$ (8). Thus, $\mathrm{CoCl}_{2}$ is a hypoxia mimetic agent, which has been frequently used for in vitro induction of hypoxia (9).

In the present study, we demonstrated that $\mathrm{CoCl}_{2}$ is well suited for the induction of hypoxia in PDA tumor xenografts growing on fertilized chicken eggs. To study therapeutic intervention with tumor hypoxia in the animal replacement model, we used the herb-derived triptolide, which is the major active substance of the vine-like Tripterygium wilfordii plant. In Chinese traditional medicine (TCM), this herb is used for the treatment of rheumatoid arthritis and cancer due to its antiinflammatory and anti-carcinogenic properties (10).

\section{Materials and methods}

Tumor cell line. The established PDA cell line BxPc-3 was obtained from the American Type Culture Collection (Manassas, VA, USA). The cells were recently authenticated by a commercial service (Multiplexion, Heidelberg, Germany). Mycoplasma-negative cultures were ensured by monthly mycoplasma tests. The cells were cultured in DMEM medium (PAA, Pasching, Austria) and supplemented with $10 \%$ heat-inactivated FCS (Sigma, Deisenhofen, Germany) and 25 mmol/l HEPES (PAA).

Treatment of cells. Triptolide (PG-490) was obtained from Sigma-Aldrich (St. Louis, MO, USA). The purity of triptolide was $98 \%$ (HPLC), and a $10 \mathrm{mM}$ stock solution was prepared in DMSO. $\mathrm{CoCl}_{2}$ was obtained from Sigma-Aldrich. The $100 \mathrm{mM}$ stock solution was prepared in phosphate-buffered saline (PBS).

Transplantation of tumor cells to the CAM of fertilized chicken eggs. This method was performed as previously described with modifications (4). Fertilized white Leghorn chicken eggs (Geflügelzucht Hockenberger, Eppingen, Germany) were incubated in a digital motor breeders Type 168/D (Siepmann $\mathrm{GmbH}$, Herdecke, Germany) at a humidity of $55-60 \%$ at $37.8^{\circ} \mathrm{C}$. The embryo with the CAM was detached at day 4 of embryonic development by removing 2-3 $\mathrm{ml}$ albumen with a syringe. Then, a small hole was cut into the eggshell, $1 \mathrm{ml}$ albumen was restituted and the hole was sealed with tape (Leukosilk; BSN Medical GmbH). At day 9 of embryonic development the tumor cells were transplanted on the CAM. Therefore small handmade rings from Thermanox ${ }^{\mathrm{TM}}$ cover discs (Thermo Scientific, Schwerte, Germany) were placed on the CAM, the CAM was scratched with a 27 gauge needle and $5 \times 10^{5} \mathrm{BxPc}-3$ were mixed 1:1 with Matrigel (BD Biosciences) and deposited into the rings. Whatman filter paper was placed to the CAM directly adjacent to the plastic ring containing the tumor cells. $\mathrm{CoCl}_{2}$, triptolide or PBS were applied via dropping to the filter paper until saturation. At day 18 , the embryos were humanely euthanized by injection of a barbiturate into the breast muscle. Tumors and the liver of the chicken embryos were resected and the tumor volumes were calculated with the formula: Volume $=4 / 3 \times \pi \times r^{3}(r=1 / 2 \times$ square root of diameter $1 \mathrm{x}$ diameter 2) (11). Tumor tissue was embedded in Tissue-Tek O.C.T. compound (Sakura, Zoeterwoude, The Netherlands) on dry ice and stored at $-20^{\circ} \mathrm{C}$.

Immunofluorescence and $H \& E$ staining. Frozen xenograft $(6 \mu \mathrm{m})$ of embryonal liver sections were fixed in ice-cold acetone at $-20^{\circ} \mathrm{C}$ for $10 \mathrm{~min}$ and blocked with $10 \%$ normal goat serum in PBS with $0.2 \%$ Tween- 20 for $30 \mathrm{~min}$ at room temperature. Incubation with primary and secondary antibodies was performed using standard procedures (1). The primary mouse monoclonal antibodies directed toward Ki67, cytokeratin 19 (both from Abcam, Cambridge, UK), EpCAM [kindly provided by G. Moldenhauer and published (12)], CD24 [(SWA11; kindly provided by P. Altevogt and published (13)], and the rabbit polyclonal antibodies carbonic anhydrase IX (CAIX; sc-25599; Santa Cruz Biotechnology, Heidelberg, Germany), CD44 (GeneTex, Nottingham, UK), Sox2 (Abcam), and CD133 (Millipore, Darmstadt, Germany) were used. The secondary antibodies were goat anti rabbit Alexa Fluor $594 \mathrm{IgG}$ or goat anti-mouse Alexa Fluor $594 \mathrm{IgG}$ (Invitrogen, Camarillo, CA, USA). Nuclei were counterstained with 4,6-diamidino-2-phenylindol (DAPI; $1 \mu \mathrm{g} / \mathrm{ml}$ ). Primary and secondary antibody was repeated with the respective antibodies. The samples were mounted in Fluoromount-G (Southern Biotech, Birmingham, AL, USA) and stored at $-20^{\circ} \mathrm{C}$ in the dark. Liver sections of chick embryo were stained with hematoxylin and eosin (H\&E; Dako, Denmark), mounted in Pro Tags Mount Aqua (Quartett, Berlin, Germany) and stored at room temperature. Representative images were captured with a Leica DMRB fluorescence microscope (Leica, Wetzlar, Germany) at x400 magnification with a SPOT ${ }^{\mathrm{TM}}$ Flex $15.264 \mathrm{Mp}$ shifting pixel digital color camera (Diagnostic Instruments; and analyzed with SPOT Basic Advanced software 4.6).

Statistical analysis. The quantitative data of chick embryo weight are presented as the means \pm SD. The tumor growth data were analyzed using Student's t-test for statistical significance. $\mathrm{P}<0.05$ was considered statistically significant. 


\section{Results}

Conventional gas hypoxia leads to rapid death of chick embryos. To study if new therapeutic options can overcome the pronounced tumor hypoxia of PDA, we tried to adapt the conventional in vitro method of gas hypoxia to chicken eggs. The eggs were placed into a hypoxia chamber, which was flushed with a gas mixture of $1 \% \mathrm{O}_{2}, 5 \% \mathrm{CO}_{2}$ and $94 \%$ $\mathrm{N}_{2}$. However, after $24 \mathrm{~h}$, even after only $30 \mathrm{~min}$, all chicken embryos were dead (data not shown). Therefore, this method of hypoxia induction is not suited for the chicken egg model.

Cobalt chloride induces expression of CAIX in PDA xenografts on fertilized chicken eggs. To study whether cobalt chloride may be more suited for induction of hypoxia in PDA xenografts on fertilized chicken eggs, we transplanted BxPc-3 cells to the CAM of fertilized chicken eggs at day 9 of incubation. At day 11, eggs with a developed and living embryo were selected and divided into 4 groups. Hypoxia was induced in half of the eggs at days $11,14,15,16$ and 17 by dropping $\mathrm{CoCl}_{2}$ until saturation to a small filter paper, which was placed directly adjacent to the tumor xenograft (Fig. 1A). Similarly, triptolide was applied via the filter paper at days 12 and 15 and the controls received PBS only. At day 18, the embryos were humanely euthanized by injection of a barbiturate into the blood vessels of CAM, followed by resection of the tumor xenografts. $\mathrm{CoCl}_{2}$ reduced the tumor volume from $0.2 \mathrm{~cm}^{3}$ in the control to $0.05 \mathrm{~cm}^{3}$ (Fig. 1B). Triptolide further reduced the tumor growth under normoxic and hypoxic conditions below 0.01 and $0.02 \mathrm{~cm}^{3}$, respectively. Most importantly, $\mathrm{CoCl}_{2}$ induced the expression of CAIX, which was measured by staining xenograft sections with a specific antibody and fluorescence microscopy (Fig. 1C). These results indicate that $\mathrm{CoCl}_{2}$ induced hypoxia-induced signaling, which was abolished by triptolide.

Cobalt chloride is well tolerated by chick embryos. In order to detect putative but unwanted side-effects of $\mathrm{CoCl}_{2}$, we analyzed developmental defects, morphology and the weight of embryos of each group. We did not observe abnormality, the embryos looked well developed and had a regular weight in each group (Fig. 2A). For detection of toxic side-effects to the liver, we stained frozen liver tissue sections with $\mathrm{H} \& \mathrm{E}$ and examined the tissue by microscopy (Fig. 2B). No necrosis was detectable, suggesting that $\mathrm{CoCl}_{2}$ did not induce toxic side-effects. Notably, triptolide alone or combined with $\mathrm{CoCl}_{2}$ did not influence development, weight or liver tissue either. Thus, $\mathrm{CoCl}_{2}$-induced hypoxia, alone or in combination with triptolide, does not induce toxic side-effects in chick embryos.

Xenograft tissue consists of human cells and proliferation is inhibited by triptolide. To ensure that the xenograft tumors consisted of human cells, we stained tumor sections with human-specific antibodies to detect proliferation by $\mathrm{Ki} 67$ and the cytoskeletal marker cytokeratin 19 (Fig. 3). Whereas both expression of cytokeratin 19 and Ki67-positive proliferating human tumor cells were detected in control and $\mathrm{CoCl}_{2}$-treated xenografts, triptolide abolished Ki67 expression, but had no effect on cytokeratin expression. This control experiment confirmed that the xenograft tissue consisted of human tumor
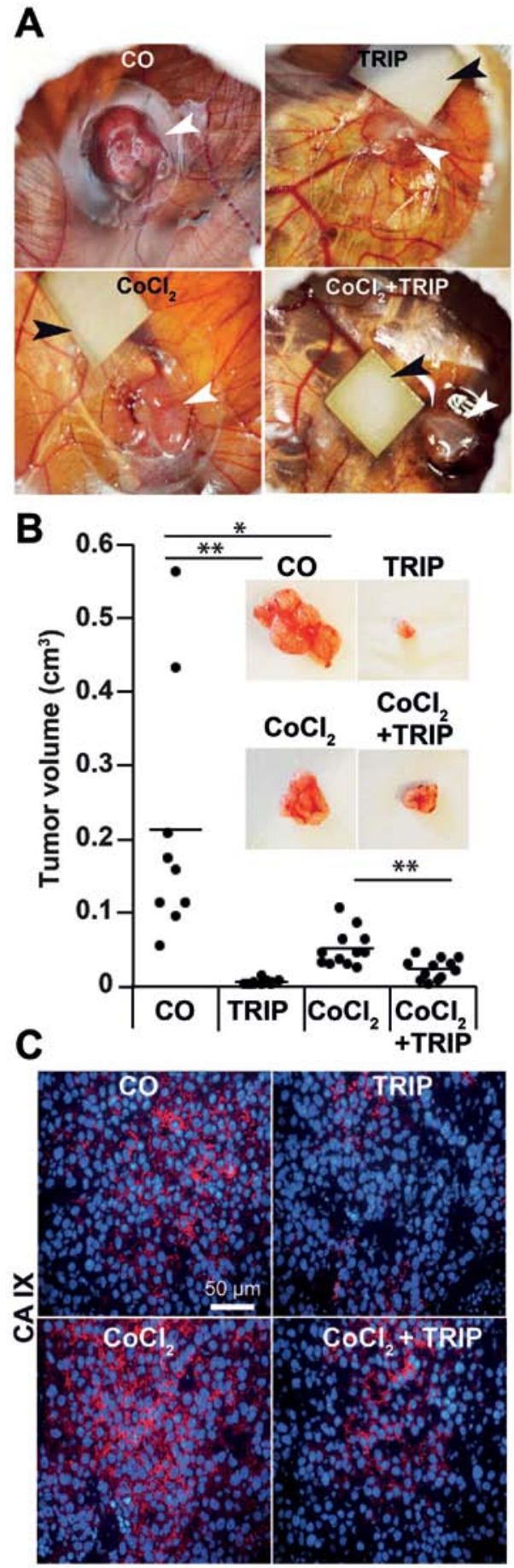

Figure 1. Cobalt chloride induces expression of CAIX in PDA xenografts on fertilized chicken eggs. (A) BxPc-3 cells $\left(5 \times 10^{5}\right)$ were transplanted in Matrigel on the CAM of fertilized chicken eggs at day 9 of embryonic development. Hypoxia was induced by dropping $10 \mu \mathrm{CoCl}_{2}(100 \mu \mathrm{M})$ to a Whatman filter paper $\left(0.5 \mathrm{~cm}^{2}\right)$ placed directly adjacent to the tumor xenografts at days 11 , $14,15,16$ and 17 until saturation. For therapeutic treatment, $10 \mu 1$ triptolide (50 nm, TRIP) was applied to the filter paper at developmental days 12 and 15 . PBS was applied as control instead of triptolide or $\mathrm{CoCl}_{2}$. White arrows point to the tumor xenografts and black bars point to the filter papers. (B) The xenografts were resected at day 18 and the tumor diameters were measured with calipers. The tumor volumes were calculated as described in Materials and methods. The volumes of the individual tumors per group are presented as black dots, and the bars indicate the average tumor size of each group. Representative images of the resected tumors are shown in the upper panel. ${ }^{* *} \mathrm{P}<0.001$; $\mathrm{P}<0.05$. (C) Sections of the xenograft tissue of each group were stained with the hypoxia marker CAIX, followed by counterstaining of nuclei with DAPI. Positive signals were detected by fluorescence microscopy. The bar indicates $50 \mu \mathrm{m}$. CAIX, carbonic anhydrase IX; PDA, pancreatic ductal adenocarcinoma; CAM, chorioallantoic membrane; $\mathrm{CoCl}_{2}$, cobalt chloride 
A

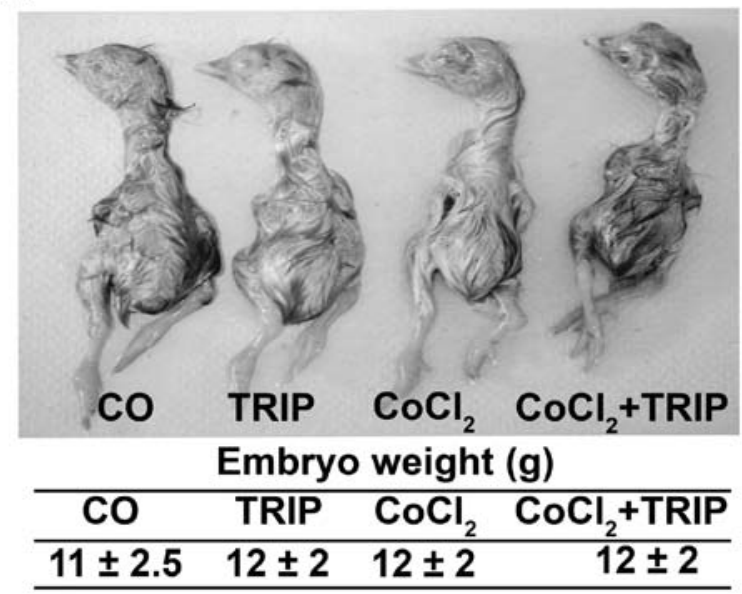

B

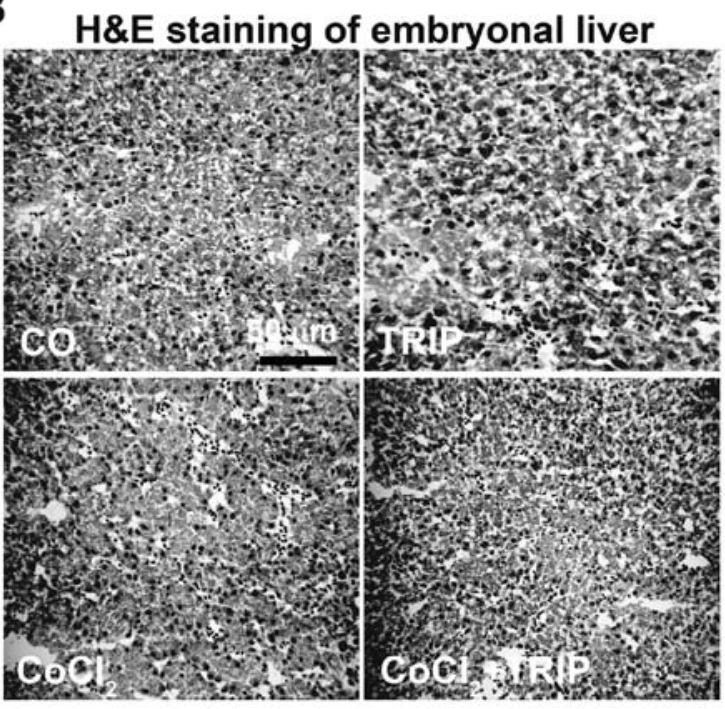

Figure 2. Cobalt chloride-induced hypoxia does not influence the weight and liver tissue of chick embryos. (A) After tumor resection, the morphology and the body weights of the chick embryos were evaluated. The average body weights of the embryos per group are presented below the pictures of representative embryos. (B) The livers of the embryos were sectioned, and $H \& E$ staining was performed and visualized under x100 magnification using microscopy. The bar indicates $50 \mu \mathrm{m}$.

cells and that $\mathrm{CoCl}_{2}$ did not decrease the proliferation, which was, however, strongly inhibited by triptolide.

Cobalt chloride induces the expression of the CSC markers CD133, Sox2, CD44 and CD24. Our recent data suggest that intrinsic tumor hypoxia and the in vitro induction of gas hypoxia increases the expression of CSC markers $(1,14)$. Therefore, we evaluated, by immunohistochemistry, whether $\mathrm{CoCl}_{2}$ may have the same effect. Indeed, we detected induction of CD133 and Sox2 (Fig. 4A) as well as induction of CD44 and $\mathrm{CD} 24$ in xenograft tissue derived from eggs with $\mathrm{CoCl}_{2}$ treatment (Fig. 4B). In contrast, triptolide alone did not induce the expression of CSC markers and triptolide co-treatment of $\mathrm{CoCl}_{2}$-treated eggs completely abolished the expression of CSC markers. These results confirm the successful induction of hypoxia in tumor xenografts on fertilized chicken eggs by application of $\mathrm{CoCl}_{2}$ to a Whatman filter paper directly adjacent to the tumor xenograft.

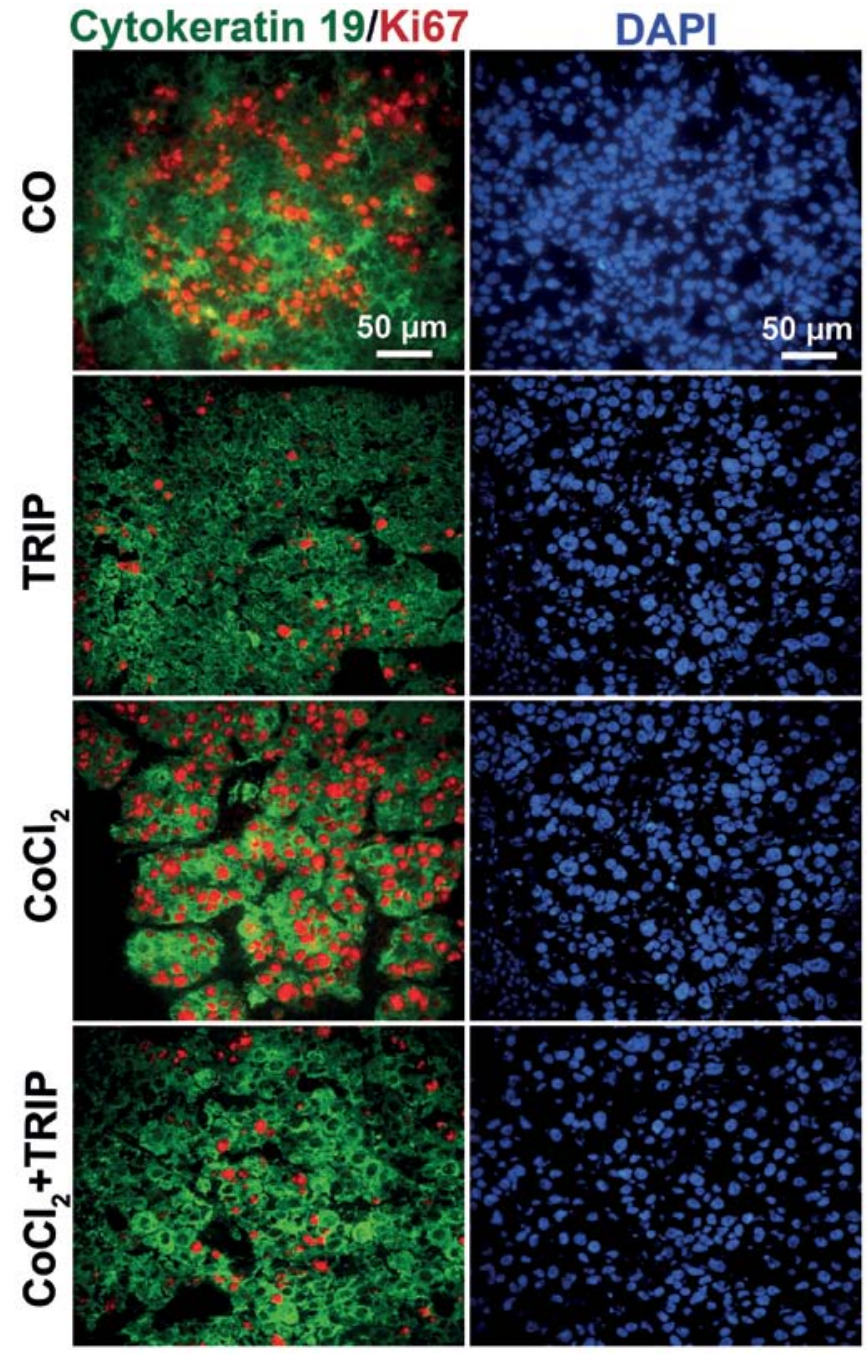

Figure 3. The human markers cytokeratin 19 and Ki67 ensure induction of hypoxia in human xenograft cells. Representative images of double immunofluorescence stainings of frozen tumor xenograft sections ( $x 400$ magnification) with the proliferation marker Ki67 (red) and the cytoskeletal marker cytokeratin 19 (Cyt19, green); both detect specifically human cells. Nuclei were stained with DAPI (blue). The bar indicates $50 \mu \mathrm{m}$.

\section{Discussion}

The aim of the present study was to establish a method for induction of hypoxia in xenografted fertilized chicken eggs without inducing toxic side-effects. However, our preliminary results demonstrated that the application of gas hypoxia is not suited as the chick embryo does not survive when eggs are incubated in a hypoxia chamber. The question is whether the induction of hypoxia in vivo is necessary, since due to the fast tumor xenograft growth, hypoxia is automatically induced, as we recently demonstrated by positive CAIX staining of PDA xenografts growing on chicken eggs (1). Although these results are confirmed by the present data, we were able to further increase the expression of CAIX and thus hypoxic signaling by $\mathrm{CoCl}_{2}$-treatment. Considering the pronounced hypoxic tumor microenvironment in patient tumors of PDA (5), this situation may reflect the tumor conditions in patients and thus may be considered as a suited model for experimental evaluation of PDA. This suggestion is underlined by our finding that 

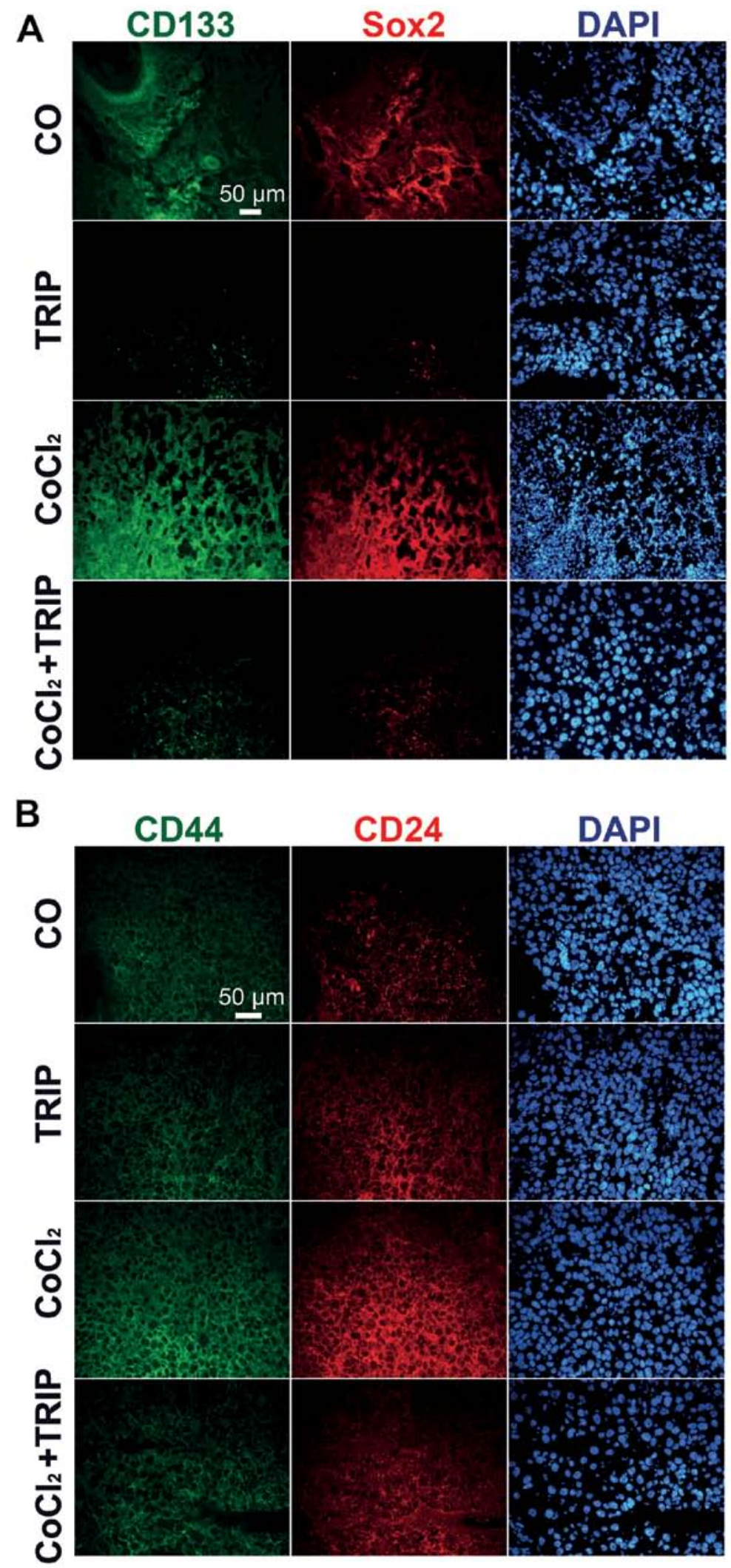

Figure 4. Cobalt chloride-induced hypoxia enhances the expression of cancer stem cell markers. (A) Representative images of immunofluorescence stainings of frozen tumor xenograft sections (x400 magnification) with the cancer stem cell markers CD133 (green) and Sox 2 (red) or (B) CD44 (green) and CD24 (red). Nuclei were stained with DAPI (blue). The bars indicate $50 \mu \mathrm{m}$.

$\mathrm{CoCl}_{2}$-induced hypoxia did not induce toxic side-effects in the chick embryo.

Recent studies suggest that hypoxia increases the aggressiveness of tumor growth (7). This finding is, however, not confirmed by the present data, since the tumor volume measured for $\mathrm{CoCl}_{2}$-treated cells was smaller compared to untreated cells. This may be due to the fact that the transplanted BxPc-3 cells are less aggressive and are therefore sensitive 
to hypoxia, as we demonstrated in our recent study (14). In the present study, in vitro gas hypoxia led to increased selfrenewal potential in highly aggressive PDA cell lines, but not in the less aggressive BxPc-3 cells. This may be due to a low percentage of CSCs, which are present in BxPc-3 cells (15). In this respect, our recent study demonstrated, that CSCs resist tumor hypoxia and start to proliferate and invade, in contrast to normal, more differentiated tumor cells $(1,14,16)$. Indeed, we observed the enhanced expression of the CSC markers CD133, Sox2, CD44 and CD24 in our model, which suggests the possibility that hypoxia induces dormant CSCs within the mixed tumor population of BxPc-3 cells.

A previous study by Lester et al (17) already used the application of $\mathrm{CoCl}_{2}$-induced hypoxia to fertilized chicken eggs transplanted with breast cancer cells. However, in this former study, the induction of hypoxia was not controlled and it was unclear if $\mathrm{CoCl}_{2}$ was effective. Also, the conditions of $\mathrm{CoCl}_{2}$ application in this former study were different from ours, since Lester et al applied $25 \mu \mathrm{l}$ of a $100 \mu \mathrm{M} \mathrm{CoCl}_{2}$ solution daily between days 9 and 18 . In contrast, our data show that a less frequent application of $\mathrm{CoCl}_{2}$ (days 11, 14, 15, 16 and 17) is sufficient for a pronounced induction of hypoxia. Our results clearly demonstrate the induction of hypoxia since we found enhanced expression of CAIX and the CSC markers Sox2, CD133, CD44 and CD24.

Finally, we demonstrated that triptolide, which was used as a treatment control, did not induce hypoxic signaling, but inhibited the $\mathrm{CoCl}_{2}$-mediated hypoxic effects. These results are in line with our previous findings, which demonstrated that triptolide strongly reverts tumor hypoxia-induced progression of PDA without inducing side-effects (1).

Taken together, $\mathrm{CoCl}_{2}$ is suitable to induce hypoxia in PDA xenografts growing on the chick embryo animal replacement model, which is a promising tool for future in vivo experimental evaluation of therapeutic strategies.

\section{Acknowledgements}

The authors thank Dr W. Gross for calculation of statistics and J. Gladkich for the technical assistance. This study was supported by grants from the German Cancer Aid (Deutsche Krebshilfe 109362), the German Research Community (DFG HE 3186/11-1), the Federal Ministry of Education and Research (BMBF 031A213), the German-Israeli Foundation for Scientific Research and Development (GIF 1058-7.11/2008), the Heidelberger Stiftung Chirurgie, the Stiftung für Krebs und Scharlachforschung, the Hanns A. Pielenz Stiftung and the Dietmar Hopp-Stiftung.

\section{References}

1. Liu L, Salnikov AV, Bauer N, Aleksandrowicz E, Labsch S, Nwaeburu C, Mattern J, Gladkich J, Schemmer P, Werner J and Herr I: Triptolide reverses hypoxia-induced EMT and stem-like features in pancreatic cancer by NF- $\kappa \mathrm{B}$ downregulation. Int $\mathrm{J}$ Cancer 134: 2849-2503, 2014.
2. Ribatti D, Nico B, Vacca A and Presta M: The gelatin spongechorioallantoic membrane assay. Nat Protoc 1: 85-91, 2006.

3. Schneiderhan W, Scheler M, Holzmann KH, Marx M, Gschwend JE, Bucholz M, Gress TM, Seufferlein T, Adler G and Oswald F: CD147 silencing inhibits lactate transport and reduces malignant potential of pancreatic cancer cells in in vivo and in vitro models. Gut 58: 1391-1398, 2009.

4. Janse EM and Jeurissen SH: Ontogeny and function of two non-lymphoid cell populations in the chicken embryo. Immunobiology 182: 472-481, 1991.

5. Brown JM and Giaccia AJ: The unique physiology of solid tumors: opportunities (and problems) for cancer therapy. Cancer Res 58: 1408-1416, 1998.

6. Hoffmann AC, Mori R, Vallbohmer D, Brabender J, Klein E, Drebber U, Baldus SE, Cooc J, Azuma M, Metzger R, Hoelscher AH, Danenberg KD, Prenzel KL and Danenberg PV: High expression of HIF1 $\alpha$ is a predictor of clinical outcome in patients with pancreatic ductal adenocarcinomas and correlated to PDGFA, VEGF, and $b F G F$. Neoplasia 10: 674-679, 2008

7. Chang Q, Jurisica I, Do T and Hedley DW: Hypoxia predicts aggressive growth and spontaneous metastasis formation from orthotopically grown primary xenografts of human pancreatic cancer. Cancer Res 71: 3110-3120, 2011.

8. Ho VT and Bunn HF: Effects of transition metals on the expression of the erythropoietin gene: further evidence that the oxygen sensor is a heme protein. Biochem Biophys Res Commun 223: 175-180, 1996.

9. Gray MJ, Zhang J, Ellis LM, Semenza GL, Evans DB, Watowich SS and Gallick GE: HIF-1 $\alpha$, STAT3, CBP/p300 and Ref-1/APE are components of a transcriptional complex that regulates Src-dependent hypoxia-induced expression of VEGF in pancreatic and prostate carcinomas. Oncogene 24: 3110-3120, 2005.

10. Brinker AM, Ma J, Lipsky PE and Raskin I: Medicinal chemistry and pharmacology of genus Tripterygium (Celastraceae). Phytochemistry 68: 732-766, 2007.

11. Balke M, Neumann A, Szuhai K, Agelopoulos K, August C, Gosheger G, Hogendoorn PC, Athanasou N, Buerger H and Hagedorn M: A short-term in vivo model for giant cell tumor of bone. BMC Cancer 11: 241, 2011.

12. Moldenhauer G, Momburg F, Möller P, Schwartz R and Hämmerling GJ: Epithelium-specific surface glycoprotein of Mr 34,000 is a widely distributed human carcinoma marker. Br J Cancer 56: 714-721, 1987.

13. Weber E, Lehmann HP, Beck-Sickinger AG, Wawrzynczak EJ, Waibel R, Folkers G and Stahel RA: Antibodies to the protein core of the small cell lung cancer workshop antigen cluster-w4 and to the leucocyte workshop antigen CD24 recognize the same short protein sequence leucine-alanine-proline. Clin Exp Immunol 93: 279-285, 1993.

14. Rausch V, Liu L, Apel A, Rettig T, Gladkich J, Labsch S, Kallifatidis G, Kaczorowski A, Groth A, Gross W, Gebhard MM, Schemmer P, Werner J, Salnikov AV, Zentgraf H, Büchler MW and Herr I: Autophagy mediates survival of pancreatic tumourinitiating cells in a hypoxic microenvironment. J Pathol 227: 325-335, 2012.

15. Kallifatidis G, Rausch V, Baumann B, Apel A, Beckermann BM, Groth A, Mattern J, Li Z, Kolb A, Moldenhauer G, Altevogt P, Wirth T, Werner J, Schemmer P, Büchler MW, Salnikov A and Herr I: Sulforaphane targets pancreatic tumour-initiating cells by NF-кB-induced antiapoptotic signalling. Gut 58: 949-963, 2009.

16. Salnikov AV, Liu L, Platen M, Gladkich J, Salnikova O, Ryschich E, Mattern J, Moldenhauer G, Werner J, Schemmer P, Büchler MW and Herr I: Hypoxia induces EMT in low and highly aggressive pancreatic tumor cells but only cells with cancer stem cell characteristics acquire pronounced migratory potential. PLoS One 7: e46391, 2012.

17. Lester RD, Jo M, Montel V, Takimoto S and Gonias SL: uPAR induces epithelial-mesenchymal transition in hypoxic breast cancer cells. J Cell Biol 178: 425-436, 2007. 